\title{
Second Language Acquisition as a Hybrid Learning Process
}

\author{
Gerard Kempen \\ NICI, University of Nijmegen, Montessorilaan 3, PO Box 9104, 6500 HE Nijmegen. The Netherlands ${ }^{1}$
}

\begin{abstract}
Recent developments in cognitive learning theory are likely to shed new light on the process of Second Language Acquisition (SLA). Basic phenomena in the behaviour of language learners suggest that SLA roots in two entirely different leaming processes: a grammatical induction device attuned to properties of natural languages, and a knowledge compilation device which according to Anderson's ACT*/PUPS model (Anderson, 1989) underlies the acquisition of cognitive skills in general. The paper presents a characterization of SLA along these lines, outlines its empirical underpinnings, and explores the implications with respect to the design of second language instruction methods and environments.
\end{abstract}

Keywords: Primary language acquisition, secondary language acquisition, second language acquisition, grammatical induction, knowledge compilation, intelligent computer-assisted language instruction.

\section{Introduction}

Recent developments in cognitive learning theory are likely to shed new light on the process of Second Language Acquisition (SLA). Basic phenomena in the behaviour of language learners suggest that SLA roots in two entirely different leaming processes: a grammatical induction device attuned to properties of natural languages, and a knowledge compilation device which according to Anderson's ACT*/PUPS model (Anderson, 1989) underlies the acquisition of cognitive skills in general. I will present a characterization of SLA along these lines, outline its empirical underpinnings, and explore the implications with respect to the design of second language instruction methods and environments.

\section{Language acquisition processes}

One of today's most prominent cognitive leaming theories is John R. Anderson's ACT* model (recently renamed PUPS; see Anderson 1989). It presupposes a distinction between three levels of analysis of cognitive mechanisms: the knowledge level, the algorithm level, and the implementation level. A knowledge level analysis describes what a person is capable of doing in principle: $\mathrm{cf}$. Chomsky's competence notion. The knowledge level abstracts away from the manner

1. Gerard Kempen is now at Department of Psychology, Leiden University, Wassenaarseweg 52, 2533 AK Leiden, The Netherlands 
in which knowledge is represented and used in actual behaviour. The algorithm level analyses how knowledge is put to use. For example, linguistic knowledge is embedded in procedures for speaking and understanding a language. Thus it specifies the actual behoviour potentials of a cognitive mochanism. (In ACT*, procedures are embodied in production systems consisting of situation-action rules whose firing is controlled by an activation-based regimen.) The observable behaviow of a cognitive mechanism depends, apart from knowledge-level and algorithmlevel determinants, on the way procedures are implemented in a computational environment. For instance, the study of speech errors typically addresses the implementation level. Linguistic performance in Chomsky's sense belongs to the algorithm or the implementation level, or to both.

Learning takes place at all three levels. At the knowledge level there is 'declarative recording' of events observed in the environment. For instance, language learners register in working memory and/or in long-term memory linguistic utterances spoken by people in their environment, together with the meanings presumably intended by these speakers. I will call these events 'intention-expression pairs' (abbreviated as I-E pairs). They form the empirical base from which children are able, by virtue of a hitherto unknown mechanism, to induce the grammar of their native language (or languages, in case children grow up in a bilingual or plurilingual environment). At any point during this primary language acquisition process, learners can test their current grammar by actually expressing their own intentions and comparing the resulting utterances with expressions which were produced by adult speakers under similar intentional conditions. In Figure 1, the two types of intention-expression pairs feeding into the grammatical induction process are designated as autogenous and allogenous I-E pairs (produced by the speaker and by the 'environment' respectively).

Leamers are helped by innate restrictions on the hypothesis space of possible natural-language grammars (cf. Pinker, 1984, 1989). This is a controversial assumption, though, rejected by various students of language acquisition including Anderson (1983). The restrictions presumably reflect 'hard-wired' properties of the Linguistic Processor. The Grammatical Induction process is located at the algorithm level: the registered declarative information is embedded in procedures (productions) which control the functioning of the Linguistic Processor.

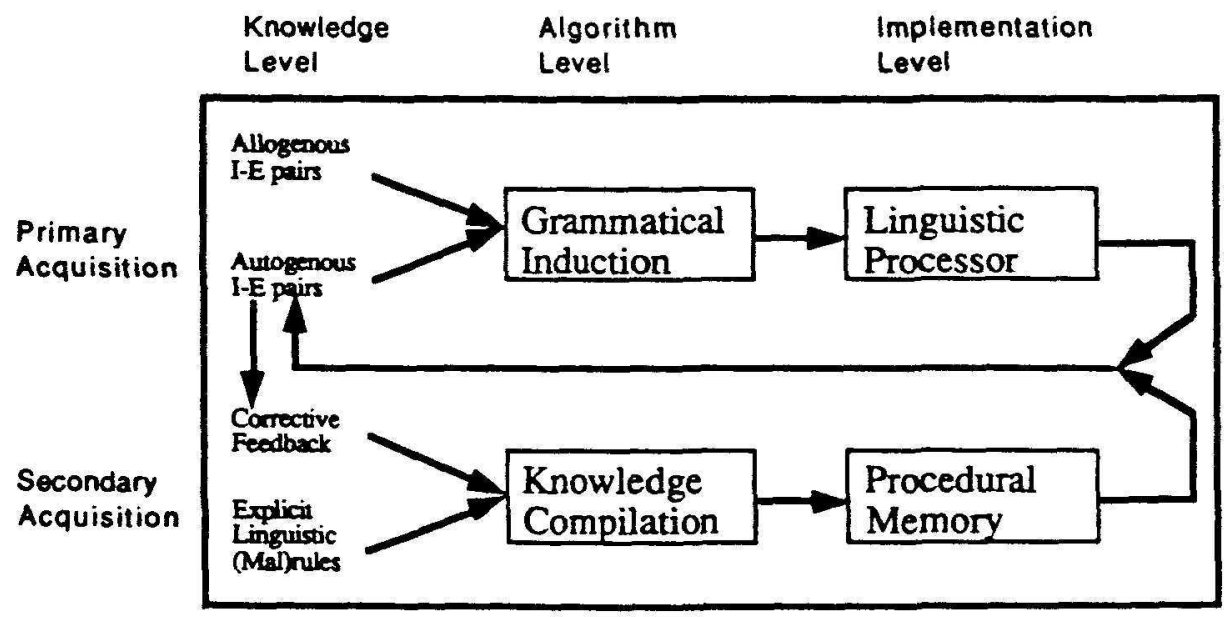

Figure 1: Two language leaming mechamisms and their interrelationships. 
Learning at the implementation level might be called 'automatization'. It does not add to the behaviour potentials of the cognitive mechanism but optimizes the value of dynamic parameters (in particular the activation levels of productions), thereby reducing the effort required to execute cognitive procedures (Anderson's term is 'strengthening'). In the context of primary language acquisition, automatization contributes to a smoother and more fluent performance of the Linguistic Processor.

In secondary or guided language acquisition the leamer starts out from explicit linguistic rules. The ACT* theory views rules of grammar as declarative knowledge which can be incorporated into procedures by a leaming process called knowledge compilation. Well-known is Anderson's application of this notion to learning command and programming languages (e.g., see Singley \& Anderson, 1989). Compilation of declarative knowledge means that a sequence of computational steps which led to the solution of a problem (c.g., applying various syntactic and morphological rules in order to determine which form of a verb is correct in the given sentential context) is collapsed into a novel one-step procedure. Confronted with new instances of the problem at later occasions, this new procedure can return the solution immediately, without having to engage in extensive problem-solving activity. The declarative grammatical knowledge which had to be retrieved from long-term memory in the course of the original solution process, has become integrated into a new procedure stored in Procedural Memory. Henceforward, when interpreting or producing a sentence in a foreign language, the leamer can rely on activating such procedural knowledge rather than on retrieving declarative knowledge in the form of explicit grammar rules. Repeated execution of compiled grammatical procedures will promote automatization and lead to higher levels of foreign language proficiency.

The process of knowledge compilation is controlled by corrective feedback about success and failure in the execution of cognitive procedures. This information is usually adduced from the environment, in particular by teachers, but can sometimes be inferred by the learners themselves (one possibility is indicated in the next section).

The assumption of specialized cognitive mechanisms for primary language acquisition is in line with Fodor's modularity thesis (Fodor, 1983) but might go against Anderson's claim that the ACT*/PUPS model is a general cognitive architecture for knowledge acquisition and still learning, i.e., encompassing both primary and secondary language acquisition. It remains to be seen whether a satisfactory account of the vast empirical differences between these language learning modes (cf. the subsequent section on primary versus secundary language acquisition) is possible within the ACT $*$ PUPS framework.

\section{The hybrid nature of second language acquisition}

I assume that primary and secondary language acquisition can work concurrently. By executing compiled grammatical procedures the leamer generates language utterances - correct or incorrect I-E pairs in the foreign language -- which can be taken into account by the Grammatical Induction process. This is indicated by the arrow from Procedural Memory to Autogenous I-E pairs in Figure 1. Secondary acquisition of a foreign language can thus trigger or catalyse primary acquisition of the same foreign language. There are effects in opposite direction as well, i.e., from primary to secondary language acquisition. Foreign language utterances constructed by the Linguistic Processor, i.e., solely on the basis of implicit rules resulting from Grammatical Induction, may serve to check correctness of utterances assembled on the basis of explicit grammar rules or compiled grammatical procedures (see the arrow from Autogenous I-E pairs to Corrective Feedback in Figure 1). 
The central new element in the proposed 'hybrid' characterization of SLA is the assumption that language users are fitted out with a double equipment. They can interpret and generate language unerances not only via the Linguistic Processor which is fed by the results of grammatical induction, but also by activating compiled grammatical knowledge in Procedural Memory. Both routes toward skilled language performance can become highly automatized. This hypothesis is at variance with alternative accounts proposed in the literature, in particular with Krashen's influential Monitor theory of SLA (Krashen, 1982), which tend to associate primary and secondary (guided) language acquisition with, respectively, automatic and controlled (i.e., nonautomatic) application of grammar rules. I think the automatic-versus-controlled distinction should be replaced by a more subtle one: the Linguistic Processor, by virtue of its being finetuned to the requirements of natural languages, is capable of attaining a higher asymptotic level of language performance than Procedural Memory. I admit that, without clarification of the nature of the said finetuning, this assumption remains purely ad hoc. However, it is in unison with various other lines of theorizing, both linguistic (universal grammar), psycholinguistic (modularity of the linguistic processor, critical period for language acquisition), and neurolinguistic (effects of left-hemisphere brain injury). I cannot go into details here; testing the proposed SLA analysis against empirical evidence is more urgent.

\section{Primary versus secondary language acquisition}

Children and young adolescents leaming a foreign language under 'total immersion' conditions can be said to learn two first languages. Their dominant mode of learning will be grammatical induction. However, second language (L2) instruction under normal classroom conditions offers limited opportunities for grammatical induction. The prevailing learning mode will therefore be knowledge compilation. The typical L2 learner produces intention-expression pairs by constructing sentences according to explicit linguistic rules. This activity leads to the compilation of these rules. The sentences themselves, being 'autogenous I-E pairs', catalyse the grammatical induction process. However, unless classroom conditions are replaced by total immersion, the role of compiled procedures in generating L2 utterances will remain prominent.

What level of L2 proficiency is attainable under such SLA conditions? The answer depends on the learner's age. There is a growing body of empirical evidence for 'maturational constraints on language leaming' (Newport, 1990): leamers starting language acquisition after the critical period, i.e., during adolescence or adulthood, end up at lower asymptotic levels of L2 performance than learners who begin during childhood. This 'fossilization' phenomenon can be explained if one assumes that the advantage of the critical period rests in the unrestrained availability of grammatical induction as a mechanism specifically geared to language acquisition, and that late starters largely rely on the less specialized knowledge compilation device.

Another well-known SLA phenomenon is free variability in L2 performance (Ellis, 1985, pp. 84 and 266). L2 leamers may produce utterances of varying grammatical quality in virtually identical speech sinuations. Ellis gives the example of a Portuguese boy learning English who produced the utterances No look my card and Don't look at my card within a few minutes of each other under very similar communicative conditions. Such observations are in keeping with the assumption of two sentence construction devices: Linguistic Processor and Procedural Memory.

The evolving L 2 competence of a foreign language learner can be described as a sequence of rule systems of increasing complexity and increasing similarity to the L2 grammar. Neither Grammatical Induction nor Knowledge Compilation would predict otherwise. However, it is a remarkable phenomenon that this sequence is more or less fixed and does not depend critically 
on similarities and differences between L2 and the learner's native language (Ellis, 1985, pp. 54-73). The proposed model can accommodate this invariance if an additional assumption is made, namely that the implicit rules discovered by the Grammatical Induction process and applied by the Linguistic Processor have a format which is different from that of compiled explicit rules, and thus are simply inaccessible to Procedural Memory (in fact, the inner workings of the Grammatical Induction mechanism are unknown).

However, would this not lead to the undesirable prediction that SLA is immune to intrusions from the mother tongue? After all, interference from the leamer's native language seems to be accountable for a non-negligible proportion of $\mathrm{L} 2$ errors. The answer is that, even without formal instruction, language learners tend to develop some explicit notions about (superficial) grammatical properties of their mother tongue, in particular conceming its lexicon and (morpho)phonology. This is declarative knowledge which at times may be hard to discriminate from the 'declarative recordings' of L2 rules (cf. Singley \& Anderson, 1989, p. 119).

Ellis also observes that, as far as spontaneous L2 production is concerned, the sequence of rule systems is virtually unaffected by the order in which rules are actually taught and by the amount of attention given to specific rule sets by the teaching method. Effects of formal instruction upon the acquisition sequence begin to manifest themselves only when the learners are focused on form, e.g., in test items (Ellis, 1985, pp. 218-224). In formal testing situations, L2 learners apparently make a stronger appeal to compiled procedures or explicit rules than during spontaneous speech where the Linguistic Processor is in control. I should add here that the empirical studies reviewed by Ellis in order to assess the effects of instruction have, for the greater par, been done with children or adolescents as L2 learners. The Grammatical Induction capability in these subjects may be assumed wholly or largely intact.

\section{Implications for the design of language instruction environments}

The SLA analysis presented here does not address the laws which govern the two main language learning processes, and therefore cannot offer detailed guidelines on how to design effective second language instruction methods and environments, computational or otherwise. What it does entail, however, is the need for two different types of teaching environment, one for each language learning mode. A teaching environment which simulates the essential feanures of 'total immersion' would be ideal for young L2 leamers who capitalize on Grammatical Induction (cf. Farrington, 1989). It should support meaningful spoken conversations about realistic events and objects. However, the current generation of Artificial Intelligence, Speech Technology, and Multimedia Presentation systems does not allow the construction of such environments (and if it did, the cost would be forbidding). Promising new developments are based on CD-ROM and CD-I (Hunziker, this volume).

Most existing software for computer assisted language instruction (CALI; e.g., see Kurtz, Chen \& Huang, 1990, Fum, Pani \& Tasso, 1991; Kempen, 1991; and Rypa's contribution to this volume) concentrates on the other learning mode by promoting the declarative recarding of explicit linguistic rules and the compilation of that knowledge. Although the technology needed to build effective systems of this kind is considerably simpler than what the simulation of total immersion requires, there is no CALI software which efficiently guides students through a nontrivial grammar fragment of a foreign language and checks the linguistic quality of their (typewritten) urterances.

In a recent survey of SLA applications of language technology, Catt \& Hirst (1990) complain about the lack of a student modelling component in nearly all CALI systems reviewed. 
They attempt to remedy this situation by building a diagnosic module which is knowledgeable about dominant types of lexical, morphological and syntactic errors made by L2 students: Interference from the leamer's mother tongue, and overgeneralizations. The heart of this module is a robust parser based on constraint relaxation techniques which is capable of analysing not only first and second language sentences but also mixed language utterances. Chanier \& Pengelly (this volume) and Fum et al. (1991) apply inference and knowledge elicitation techniques from Artificial Intelligence in the prototype student models they designed. Lehman (this volume) develops an adaptive parser which is sensitive to the errors of individual language leamers.

The ultimate CALI systems of the future will be able to integrate, or at least to switch between, the 'tocal immersion' and 'explicit grammar' modes of language leaming while taking into account student preferences, results of error diagnosis, and knowledge about the optimization of primary and secondary language acquisition. I am aware that building such systems cannot be a short-term goal.

\section{References}

Anderson, JR. (1983). The Anchitecure of Cognition. Cambridge, MA: Harvard University Press.

Anderson, J R. (1989). A theary of the origins of human knowledge. Artificial Inielligence, 40. Also in: J.G. Carbonell (Ed.). Machine Leaming: Paradigms and Methods. Cambridge, MA: MIT Press.

Catt, M., \& Hirst, G. (1990). An intelligent CALI system for grammatical error diagnosis. Computer Assisted Language Learning, 3, 3-26.

Chanier, T., \& Pengelly, M. (this volume). Knowledge acquisition for learner madelling in second language learning. Paper presented at the NATO Advanced Research Workshop on 'Cognitive Modelling and Interactive Environments', Mierlo, The Netherlands.

Ellis, R. (1985). Under standing Second Language Acquisition. Oxford: Oxford University Press.

Farnington, B. (1999). Al: 'Grandeur' or 'Serviude'? In: K. Cameron (Ed.). Computer Assisted Language Learning. Oxford: Intellect Books.

Fodor, J.A. (1983). The Madularity of Mind. Cambridge, MA: MTT/Bradford Books.

Fum, D., Pani, B, Tasso, C. (1991). Teaching the English tense: Integrating naive and formal grammar in an inelligent wer for foreign language teaching. In: Proceedings of the Fifh Conference of the European Chapter of the Association of Computational Linguistic (Berlin).

Hunziker. H.W. (this volume). Modular prototyping in the design of interactive learning programs. Paper presented at the NATO Advanced Research Workshop on 'Cognitive Modelling and Interactive Environments', Mierlo, The Netherlands.

Kempen, G. (1991). Lenguage technology and language instruction: Computational diagnosis of word-level errors. In: M. Swart a M. Yazdani (Eds). The Bridge to International Communication: Intelligent Tutoring Systems for Second Language Learning. New York: Springer-Verlag.

Krashen, S. (1982). Principles and Practice in Second Language Acquisition. Oxford: Pergamon Press.

Kurtz, B.L., Chea, L., \& Huang, X-M. (1990). An English-Chinese language learning system using adaptive correction and multipass error diagnosis. Journal of Artificial Intelligence in Education, 1, 51-64.

Lehman, J. Fain (this volume). Three uses of adaptive parsing in intelligent nutoring. Paper presented at the NATO Advanced Reserch Workshop on 'Cognitive Modelling and Interactive Environments', Mierlo, The Netherlands.

Newport, E. (1990). Maturational constraints on language leaming. Cognitive Science, 14, 11-28.

Pinker, S. (1984). Language Leamability and Language Development. Cambridge, MA: Harvard University Press.

Pinker, S. (1989). Language acquisition. In: D.N. Osherson \& H. Lasnik (Eds). An Invitation to Cognitive Science. Vol. 1: Language. Cambridge, MA: MIT Press.

Rypa, M. (this volume). CALLE: A computer-assisted language learning environment. Paper presented at the NATO Advanced Research Workshop on 'Cognitive Modelling and Interactive Environments', Mierlo. The Necherlands.

Singley, M.K. \& Anderson, J.R. (1989). The Transfer of Cognitive Skill. Cambridge, MA: Harvard University Press. 